\title{
Nueva especie del quitón Lepidochitona (Polyplacophora: Ischnochitonidae) de Puerto Rico
}

\section{Cedar I. García-Ríos}

Departamento de Biología, Universidad de Puerto Rico en Humacao, Humacao, Puerto Rico 00792;

cedar.uprh@gmail.com

\author{
Recibido 29-VI-2009. Corregido 20-X-2009. Aceptado 25-XI-2009.
}

\begin{abstract}
A new species of the chiton Lepidochitona (Polyplacophora: Ischnochitonidae) from Puerto Rico. The genus Lepidochitona Gray, 1821 has two species in the Caribbean: L. liozonis (Dall \& Simpson, 1901) and L. rosea Kaas, 1972. We describe a third species: Lepidochitona rufoi sp. nov. from Puerto Rico. Lepidochitona rufoi differs from both in having a finely granulose tegmentum, smaller size, a very fine central radula tooth, and different anal plate form and color pattern. It also differs from $L$. liozonis in having longer marginal spicules. Rev. Biol. Trop. 58 (2): 635-644. Epub 2010 June 02.
\end{abstract}

Key words: Mollusca, Polyplacophora, Lepidochitona, Puerto Rico, new species.

El género Lepidochitona Gray, 1821 se encuentra representado en el Caribe por dos especies: L. liozonis (Dall \& Simpson, 1901) y L. rosea Kaas, 1972 (Kaas \& Van Belle 1985, Slieker 2000). Lepidochitona liozonis fue la primera en ser descrita, el Holotipo lo recolectaron en la Isla de Culebra, Municipio de Puerto Rico (Dall \& Simpson 1901). La segunda especie, L. rosea, fue descrita a partir de dos ejemplares en mal estado (Kaas 1972). Siendo el Holotipo, con sus seis primeras valvas rotas, con lo que se cuenta del material de referencia de la descripción original.

El estudio de un lote de quitones recolectado en Tobago en 2007 reveló la presencia de una docena de ejemplares que corresponden a la descripción de L. rosea (García-Ríos \& Álvarez-Ruiz 2008). Tobago es la isla vecina de Trinidad que es la localidad del Holotipo de L. rosea. Las observaciones del tegumento y los elementos del cinturón, por medio de imágenes de microscopía electrónica de barrido, de los ejemplares de Tobago, permitió diferenciar esta especie de la ilustrada en García-Ríos (2003) como L. rosea. Los dos ejemplares, erróneamente identificados en García-Ríos (2003), pertenecen a una especie nueva, que se describe a continuación.

\section{MATERIALES Y MÉTODOS}

Cuatro ejemplares de L. rufoi fueron recolectados en Ensenada Yegua, cerca de la Reserva Natural Las Cabezas de San Juan, Fajardo, Puerto Rico (18 23'06'N-65'37'37'O), entre $1-2 \mathrm{~m}$ de profundidad, próximo a la cresta del arrecife de borde. Tres de los ejemplares fueron encontrados sobre las conchas del gasterópodo Astraea tuber Linné, 1758. El otro fue encontrado asociado al alga verde calcárea Halimeda discoidea Decaisne, 1842. Los cuatro ejemplares fueron preservados en etanol al $70 \%$.

Los datos merísticos de los quitones se realizaron usando un microscopio de disección (Nikon SMZ1500) con un micrómetro en el ocular. Los colores se describieron comparando los ejemplares preservados con la guía de 
colores de Smithe (1975). Los dos ejemplares seleccionados para las observaciones de la rádula, los elementos del cinturón y la micro topografía del tegumento, se prepararon utilizando el método descrito en Bullock (1985). Los tejidos blandos de los quitones se disolvieron usando una solución caliente de $\mathrm{KOH}$ $2 \mathrm{~N}$. Las valvas que presentaban costras calizas sobre su superficie, fueron tratadas con ultrasonido por uno o dos minutos. Las estructuras ya limpias se lavaron con agua destilada y, finalmente, se almacenaron en etanol al 95\%.

Las estructuras seleccionadas para preparar las fotografías, utilizando el microscopio electrónico de barrido (MEB), se secaron al aire y se fijaron en bases de aluminio usando un adhesivo de carbón. Estos ejemplares se cubrieron con una película de oro usando un evaporador.

La determinación de la densidad de estetos se realizó utilizando fotografías de la superficie del tegumento con magnificaciones de 300X. Sobre las fotos se dibujaron cuadrantes de 150x $150 \mu \mathrm{m}$ y se realizó el conteo de los poros que corresponden a la posición de los macro y microestetos.

Los elementos del cinturón se encuentran embebidos en una base de quitina transparente, opaca para el MEB, pero visibles en el microscopio de luz. Para determinar las dimensiones y colores de esos elementos se utilizó un microscopio compuesto (Olympus BH-2) con gradilla calibrada para medir. Todas las medidas de las estructuras expuestas se corroboraron con las fotografías del MEB.

Los ejemplares tipo fueron depositados en el Museo de Zoología Estatal de Munich (Zoologische Staatssammlung Manchen, ZSM), Alemania y la Colección Biológica de la Universidad de Puerto Rico en Humacao (CBUPRH).

Sistemática: Según la clasificación de Kaas \& Van Belle (1998):
Clase POLYPLACOPHORA Gray, 1821

Orden NEOLORICATA Bergenhayn, 1955

Suborden ISCHNOCHITONINA

Bergenhayn, 1930

Familia ISCHNOCHITONIDAE Dall, 1889

Subfamilia LEPIDOCHITONINAE

Iredale, 1914

Género Lepidochitona Gray, 1821

Subgénero Lepidochitona s.s.

Lepidochitona (L.) rufoi García-Ríos, sp. nov.

(Figuras 1-7)

\section{MATERIAL EXAMINADO}

Holotipo: ZSM Mol 20090397: Ejemplar completo, preservado en etanol, $4 \mathrm{~mm}$ de longitud. Puerto Rico, Fajardo, Seven Seas, Ensena-

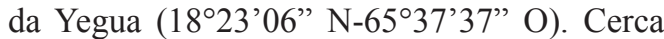
de la cresta del arrecife; $2 \mathrm{~m}$ de profundidad, sobre conchas del gasterópodo A. tuber. Recolectado por el autor, 17 de julio de 2000.

Paratipos: ZSM Mol 20090398: Ejemplar completo, preservado en etanol, $3 \mathrm{~mm}$ de longitud. Misma localidad que el Holotipo, pero encontrado asociado al alga verde calcárea $H$. discoidea, $1 \mathrm{~m}$ de profundidad. Recolectado por Rufo M. Vega Pagán, 7 de marzo de 1985; CBUPRH \#1878, 4mm de longitud (incluyendo el cinturón), la misma localidad e información de colección que el Holotipo, desarticulado; CBUPRH \# 1997, 3.5mm longitud (incluyendo el cinturón), la misma localidad e información de colección que el Holotipo, desarticulado.

\section{ENGLISH DIAGNOSIS OF NEW TAXON}

Oval, small chiton up to $4 \mathrm{~mm}$ long when stretched (Figs. 1 and 2). Width approximately half of the length. Dorsal elevation of plate IV is 0.28 (CBUPRH \#1878); back rounded, not 


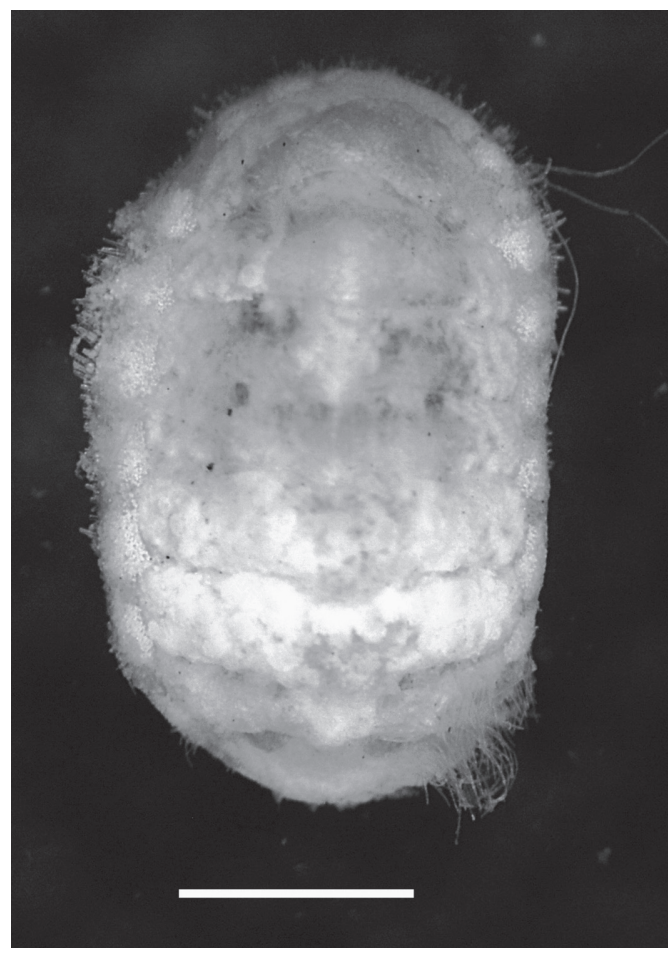

Fig. 1. Lepidochitona rufoi García-Ríos, sp. nov. Holotipo, ZSM Mol 20090397, longitud de la escala $=1 \mathrm{~mm}$.

Fig. 1. Lepidochitona rufoi García-Ríos, sp. nov. Holotype, ZSM Mol 20090397, scale bar=1mm.

carinated; side slopes a little convex to straight. Color of tegmentum cream with irregular ochre yellow marks. The marks are more abundant near the jugal ridge, in the pleural areas. Three of the four specimens with calcareous incrustations and algal growth in the tegmentum.

Anterior margin of head valve semicircular; posterior margin forming a $135^{\circ}$ angle with a rounded notch in the middle. Intermediate valves rectangular, the posterior margin concave on both sides of the apex (Figs. 3B and C). Apex well defined in all the intermediate valves. From valve III to VII the front edge is a little depressed between apophyses. Lateral areas are not raised, and their separation from the central area is not well defined. The anal valve is smaller than the head valve (Fig. 3D). The tegmentum of the anal valve is 2.5 times

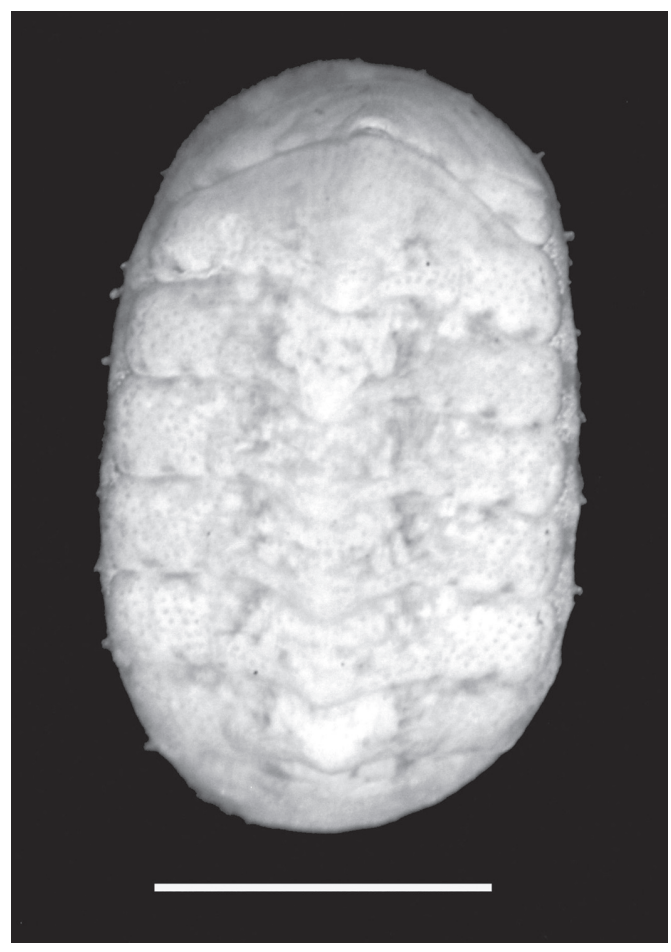

Fig. 2. Lepidochitona rufoi García-Ríos, sp. nov. Paratipo, ZSM Mol 20090398, longitud de la escala $=1 \mathrm{~mm}$.

Fig. 2. Lepidochitona rufoi García-Ríos, sp. nov. Paratype, ZSM Mol 20090398, scale bar=1 mm.

wider than long. It has a semicircular posterior edge (approximately a third of a circle); the anterior edge is convex. Mucro not prominent, anterior, in the first third of the length. The post-mucronal slopes almost straight, a little concave (Fig. 4).

The tegmentum at plain sight looks smooth, but porous under the dissecting microscope. The SEM reveals a microgranular surface, with irregular granules $50 \mu \mathrm{m}$ in diameter, well formed in the anterior margin of the central area (Fig. 5) and the exterior borders of the lateral area of intermediate valves. In the head valve the granules are absent near the apex. The tail valve granules appear in the post-mucronal area, distal from the mucro (Figs. 3D and 4).

Each granule is arranged by an elevated solitary megaesthete in its center, with more 


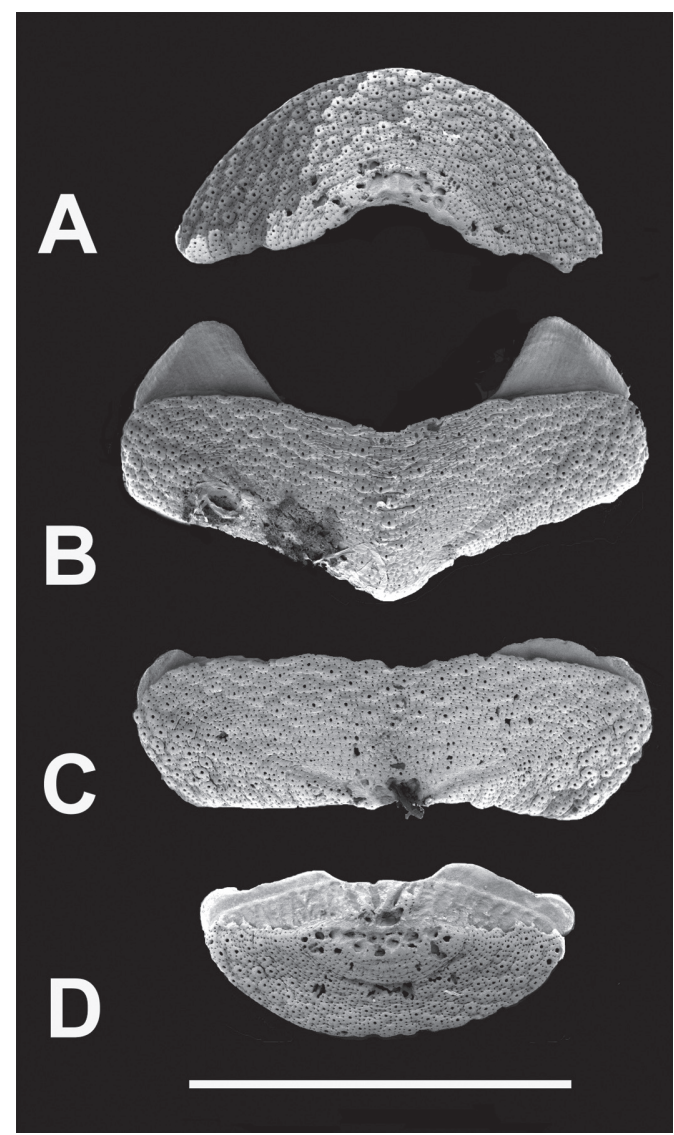

Fig. 3. Lepidochitona rufoi García-Ríos, sp. nov. Longitud total del ejemplar $3.5 \mathrm{~mm}$, incluyendo el cinturón, Paratipo CBUPRH \#1997; vista dorsal de: (A) valva cefálica; (B) valva II; (C)valva IV; (D)valva anal; longitud de la escala $=1 \mathrm{~mm}$.

Fig. 3. Lepidochitona rufoi García-Ríos, sp. nov. Whole specimen $3.5 \mathrm{~mm}$ long including girdle, Paratype CBUPRH \#1997; dorsal view of: (A)head valve; (B)valve II; (C) valve IV; (D)tail valve; scale bar $=1 \mathrm{~mm}$.

or less 10 microesthethes in the periphery; other microesthethes are present between the granules (Fig. 5). In the central area there are around 310 megaesthethes $/ \mathrm{mm}^{2}$ and 3800 to 4200 microesthethes $/ \mathrm{mm}^{2}$; with an average proportion of $1: 13$. The density of the esthetes is independent of the presence and definition of the granules. Esthetes are arranged in longitudinal rows on the central area and in radiating rows on the lateral areas.
The articulamentum is translucent, with a whitish tint. The insertion plate is short, with slit formula 10/1/9 (CBUPRH \#1878). The teeth on the insertion plate are irregular; slits rays visible. Apophyses are triangular in valve II (Fig. 3B) and trapezoid in posterior valves. The jugal sinus is ample, approximately a third of the valve width (Fig. 3B and C).

Girdle is colored like the tegmentum, in alternating bands. Dorsally it is clothed with closely set, non imbricating cylindrical corpuscules, colored, and with rounded heads (Fig. 6A). Many of the cylindrical corpuscules show fine grooves. These corpuscules have diameters of $11 \mu \mathrm{m}$ and lengths of $20-40 \mu \mathrm{m}$, but mostly close to $30 \mu \mathrm{m}$. Curved spicules, which can reach lengths of up to $70 \mu \mathrm{m}$ (chiefly broken), occur near the sutures at mid distance between the tegmentum and the outer margin of the girdle. The marginal spicules are up to $112 \times 15$ $18 \mu \mathrm{m}$, obtuse and transparent (Fig. 6B). These marginal spicules are a little flattened in the ventral face and have very fine striate in the upper surface. In contact with the marginal spicules, in the ventral side, there is a row of submarginal scales, flat, almost rectangular, of about $60 \times 20 \mu \mathrm{m}$ (Fig. 6C). The undersurface of the girdle is paved with pointed, imbricating, translucent scales, about 30-40x9-10 $\mu$ m. These scales are oriented with the pointed end toward the margin; the ventral face is a little flattened and the dorsal side arched (Fig. 6D).

Central tooth of radula very narrow, width less than $5 \mu \mathrm{m}$, and sharply pointed. Major lateral tooth tricuspid (Fig. 7). Gills merobranchial, abanal, with 0 ctenidia on each side in specimens of 3 to $4 \mathrm{~mm}$ long.

\section{Descripción de la especie}

Quitón ovalado y pequeño, hasta $4 \mathrm{~mm}$ de longitud total cuando está extendido (Figs. 1 y 2). Ancho es aproximadamente la mitad de la longitud. Elevación dorsal de la valva IV es de 0.28 (ejemplar CBUPRH \#1878); dorsalmente redondeado, sin quilla en la región del yugo. Pendiente lateral casi recta, ligeramente convexa. Tegumento de color crema claro con 


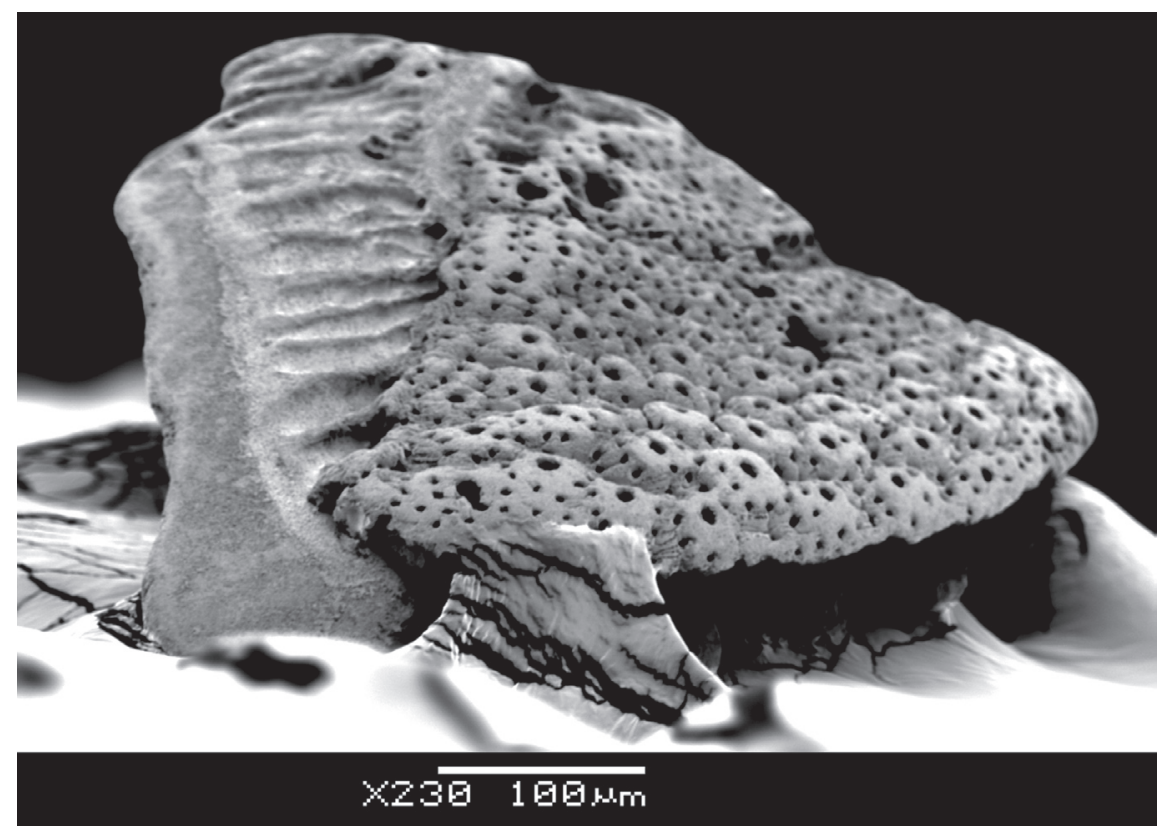

Fig. 4. Lepidochitona rufoi García-Ríos, sp. nov., Paratipo CBUPRH \#1997: vista lateral de la valva anal.

Fig. 4. Lepidochitona rufoi García-Ríos, sp. nov., Paratype CBUPRH \#1997: lateral view of tail valve.

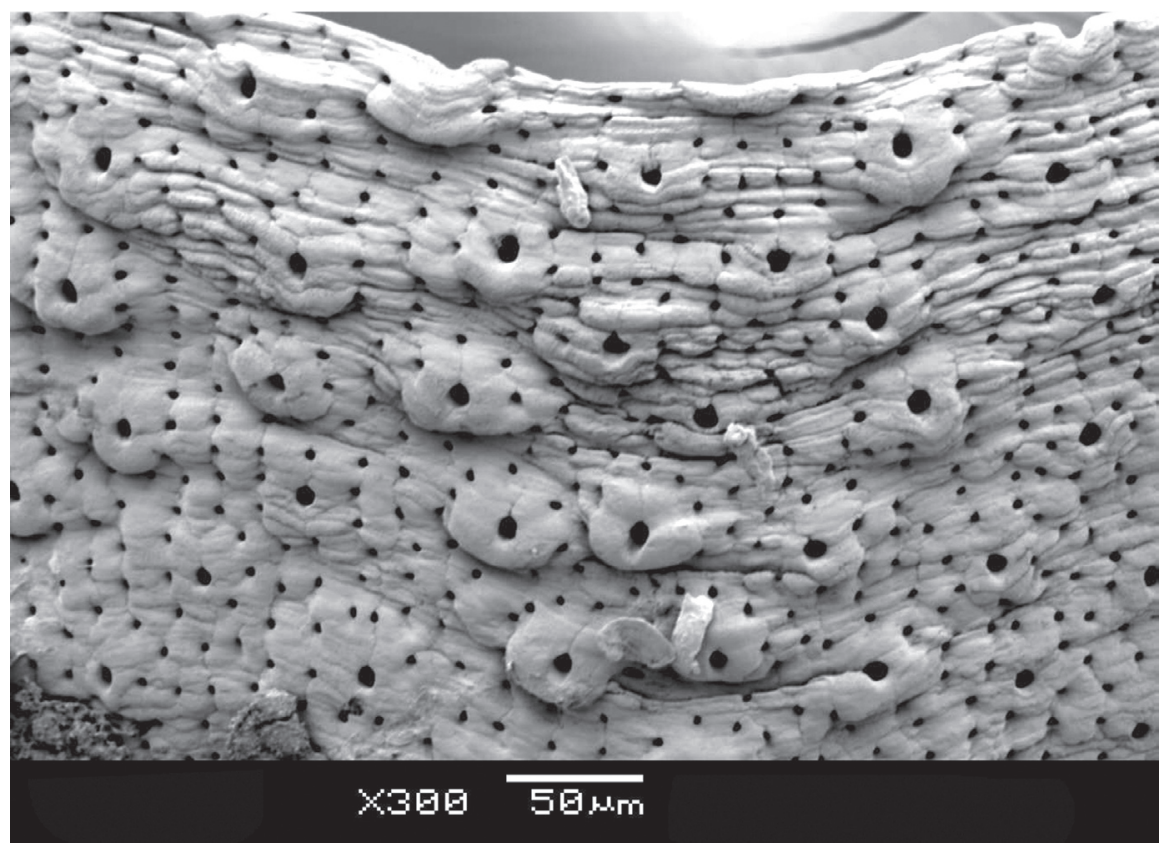

Fig. 5. Lepidochitona rufoi García-Ríos, sp. nov., Paratipo CBUPRH \#1997: detalle del tegumento en el área central de la valva II.

Fig. 5. Lepidochitona rufoi García-Ríos, sp. nov., Paratype CBUPRH \#1997: detail of central area of tegmentum of valve II. 


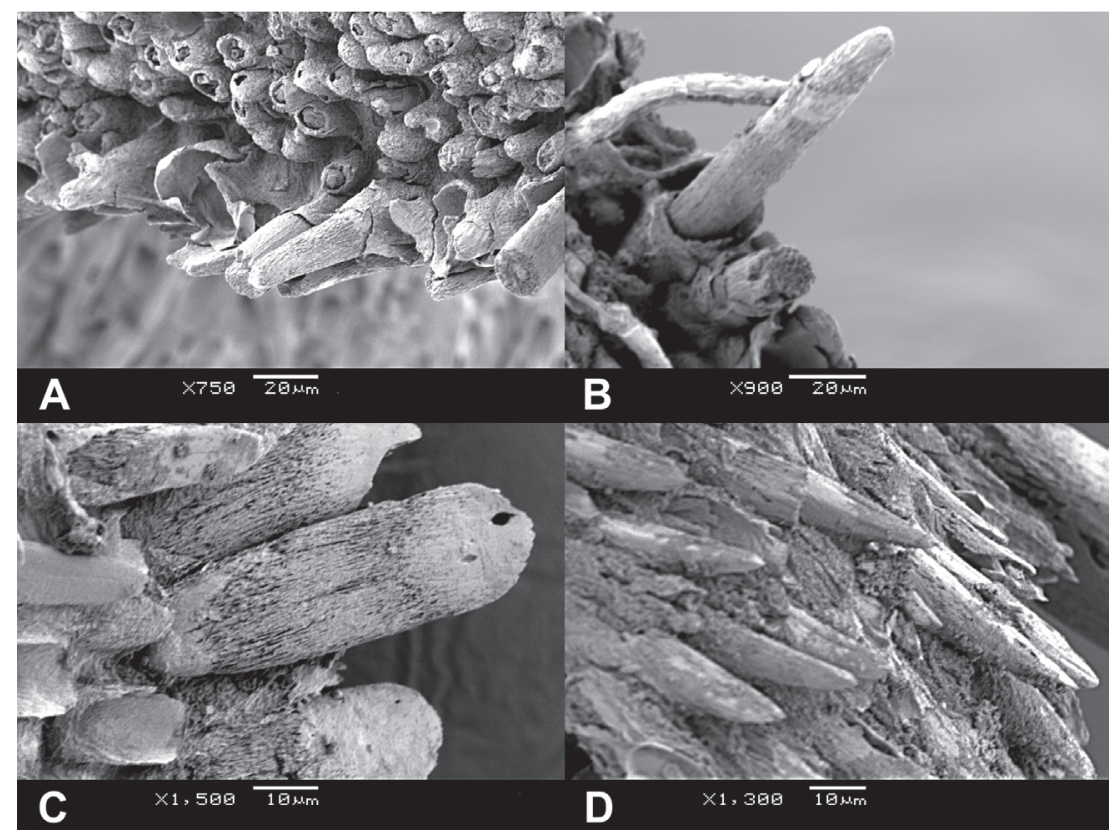

Fig. 6. Lepidochitona rufoi García-Ríos, sp. nov. Longitud total del ejemplar 4mm, incluyendo el cinturón, Paratipo CBUPRH \#1878. Cinturón: (A)vista dorsal del borde; (B)espícula marginal; (C)espícula submarginal; (D)espículas ventrales.

Fig. 6. Lepidochitona rufoi García-Ríos, sp. nov. Whole specimen 4mm long including girdle, Paratype CBUPRH \#1878. Girdle: (A)dorsal view of border; (B)marginal spicule; (C)submarginal spicule; (D)ventral spicules.

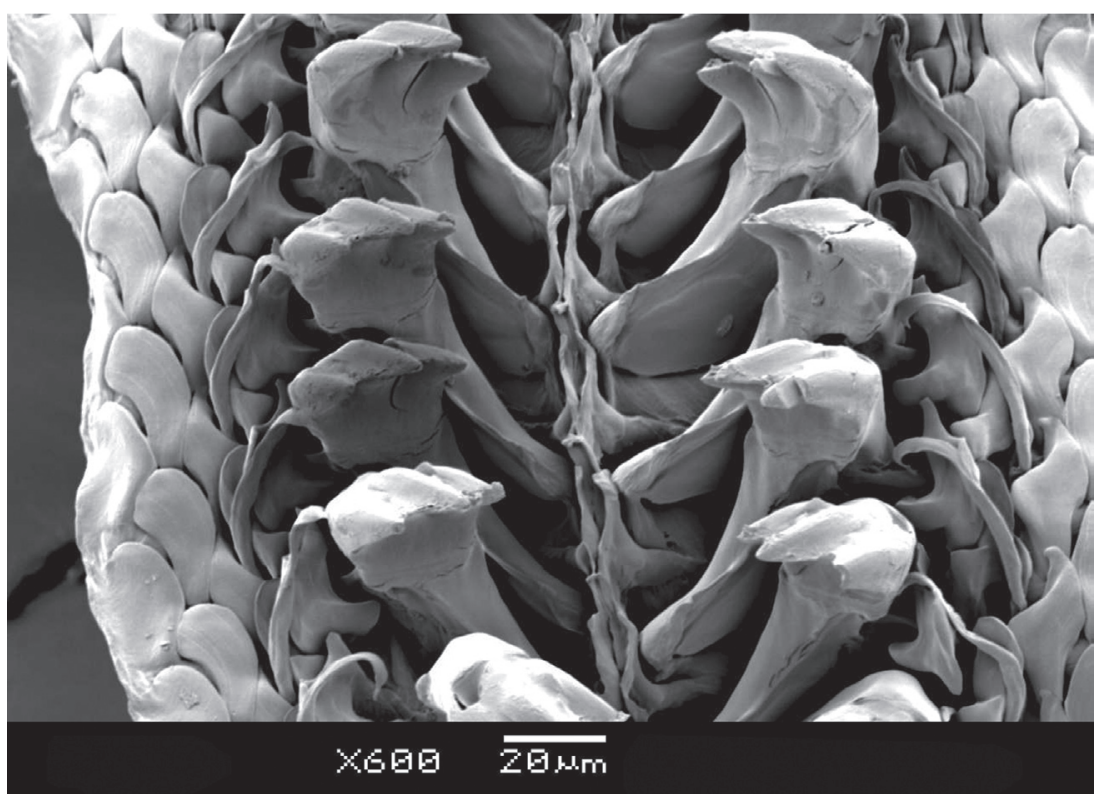

Fig. 7. Lepidochitona rufoi García-Ríos, sp. nov., Paratipo CBUPRH \#1997: rádula.

Fig. 7. Lepidochitona rufoi García-Ríos, sp. nov. Paratype CBUPRH \#1997: radula. 
marcas irregulares de coloraciones amarillo ocre: las marcas son más abundantes en los flancos de la zona del yugo, que en el área pleural. Tres de los cuatro ejemplares presentan costras calcáreas y algas filamentosas que cubren porciones del tegumento (Fig. 1).

Valva cefálica con el borde anterior semicircular, casi completa medio círculo (Fig. 3A). El borde posterior forma un ángulo de $135^{\circ}$ y presenta una muesca redondeada en el centro. Valvas intermedias rectangulares, con el margen posterior semi cóncavo a ambos lados del ápice (Fig. 3B y C). Ápice bien definido en todas las valvas intermedias. De la valva III - VII el borde anterior está ligeramente deprimido entre apófisis. Las áreas laterales no están elevadas y no está bien definida su separación con respecto del área central. Valva anal más pequeña que la cefálica (Fig. 3D) y presenta tegumento 2.5 veces más ancho que largo, así como un borde posterior semicircular (aproximadamente un tercio de círculo) y el borde anterior convexo. El mucrón no es prominente, localizado anteriormente hacia el primer tercio de la longitud de la valva anal. La pendiente postmucronal es casi recta, semi-cóncava (Fig. 4).

Tegumento es liso a simple vista; de apariencia porosa bajo el microscopio de disección. Las imágenes del MEB revelan una superficie microgranular, donde los gránulos irregulares que promedian $50 \mu \mathrm{m}$ de diámetro, están mejor definidos hacia los bordes anteriores del área central (Fig. 5) y los bordes del área lateral de las valvas intermedias. En la valva cefálica los gránulos están ausentes cerca del ápice y aumentan su tamaño cerca del borde cercano al cinturón. En la valva anal los gránulos aparecen en el área postmucronal, distales con respecto del mucrón (Fig. 3D y 4). Los gránulos tienen en su extremo más alto un megaesteto y en la periferia de 8-10 microestetos, los microestetos también se encuentran entre los gránulos (Fig. 5). En el área central se encuentran 310 megaestetos $/ \mathrm{mm}^{2}$ y entre 3800 y 4200 microestetos/ $\mathrm{mm}^{2}$; una razón promedio de 1:13. La densidad de los estetos es independiente de la presencia y definición de los gránulos. Los estetos están alineados longitudinalmente en el área central y radialmente en las áreas laterales.

Articulamento traslúcido, con un tinte blanquecino, que permite ver los colores del tegumento. Placa de inserción es corta, con fórmula de hendiduras de inserción de 10/1/9 (ejemplar CBUPRH \#1878). Dientes de la placa de inserción de tamaño irregular, radios de las hendiduras están presentes. Apófisis se eleva en punta en la valva II (Fig. 3B) y con bordes más aplanados en las valvas posteriores. Apófisis se encuentran bien separadas, dejan un amplio seno del yugo de aproximadamente un tercio del ancho de la valva (Figs. 3B y C).

El cinturón de L. rufoi tiene los mismos colores que el tegumento, presentando bandas alternas en tonalidades claras y oscuras. Dorsalmente está cubierto con corpúsculos cilíndricos en un arreglo compacto, pero no solapados, con los extremos redondeados y se pueden distinguir estrías en el extremo expuesto (Fig. 6A). Estos corpúsculos tienen diámetros de $11 \mu \mathrm{m}$ y longitud variada, de 20 a $40 \mu \mathrm{m}$, la mayoría de $30 \mu \mathrm{m}$. Los más largos están localizados en el borde interior, en contacto con el área de unión de las valvas. Cerca de las suturas, a media distancia entre el tegumento y el margen exterior del cinturón, destacan espículas curvas, con longitudes que pueden alcanzar los $70 \mu \mathrm{m}$, desafortunadamente la mayoría de ellas no están completas. En el margen del cinturón destacan largas espículas, hasta $112 \mu \mathrm{m}$ y de 15 a $18 \mu \mathrm{m}$ de diámetro en la base, son obtusas y transparentes (Fig. 6B). Ventralmente las espículas marginales son achatadas y dorsalmente presentan estrías poco profundas. Adyacentes a las espículas marginales se encuentra una fila de escamas submarginales achatadas, semi rectangulares y de punta roma, de unos $60 \mu \mathrm{m}$ de longitud y $20 \mu \mathrm{m}$ de ancho (Fig. 6C). Ventralmente el cinturón está cubierto por escamas transparentes de unos $30-40 \mu \mathrm{m}$ de largo, alrededor de 9 a $10 \mu \mathrm{m}$ en la base y con punta pronunciada, orientadas hacia el margen, y su parte ventral achatada y dorsalmente cóncava (Fig. 6D). 
Rádula con diente central delgado, hasta 5 $\mu \mathrm{m}$ de ancho y de punta afilada. Diente lateral mayor tricúspide (Fig. 7).

Las branquias se distribuyen a partir de la región media de la valva III y se extienden hasta el borde posterior de la valva VI (merobranquial). En ejemplares de 3 a $4 \mathrm{~mm}$ de longitud se observaron 10 branquias a cada lado a lo largo del surco palial. El tamaño de las branquias aumenta posteriormente (abanal).

Etimología: Esta especie está dedicada al biólogo marino Rufo M. Vega Pagán, encargado de las actividades subacuáticas del Programa de Biología Marina Costanera en la Universidad de Puerto Rico en Humacao, recolector del primer ejemplar de esta especie en nuestra colección, considerado como paratipo en este estudio.

\section{DISCUSIÓN}

La clasificación de esta especie en el género Lepidochitona responde principalmente a las características de los elementos de su cinturón, cubierto con corpúsculos que no se sobreponen. En forma similar, el subgénero Lepidochitona tiene un cinturón con presencia de espículas hialinas reducidas y sin ramificar, diferente al subgénero Dendrochiton Berry, 1911, que presenta procesos córneos ramificados. La especie que aquí se describe presenta una hendidura a cada extremo de las valvas intermedias, a diferencia de los lepidoquitones del subgénero Spongioradsia Pilsbry 1894, que presenta múltiples hendiduras en las placas de inserción de las valvas intermedias.

Esta especie se encuentra entre las más pequeñas que se conocen. De las 20 especies reportadas para el subgénero Lepidochitona $s$. $s$. reseñadas en Kaas \& Van Belle (1985) sólo tres tienen longitudes iguales o menores a 5mm: L. iberica Kaas \& Van Belle, 1981; L. turtoni (Ashby, 1928) y L. subaleutica (Sirenko, 1976). De esa lista, los dos lepidoquitones más pequeños corresponden a las dos especies de más reciente descripción. La descripción relativamente reciente de estas especies, como la que aquí se presenta, puede responder a la dificultad de recolectar y reconocer ejemplares pequeños. Estas especies pequeñas presentan las mayores elevaciones dorsales, desde 0.39 a 0.66. En contraste, la elevación dorsal de L. rufoi es menor, si se le compara con las 19 especies para las que existe ese dato (Kaas \& Van Belle 1985), solamente se reportan valores menores en cuatro especies. Esta combinación de tamaño reducido y poca elevación dorsal, sumado al crecimiento de algas epizooicas sobre sus valvas, hacen que esta especie sea poco conspicua.

Los colores dominantes en las especies del Caribe son el rojo oscuro en L. liozonis (Dall \& Simpson 1901, Abbott 1974) y rojo-rosado en L. rosea (Kaas 1972, Slieker 2000). Se registran bandas blancas y marcas crema, marrones y hasta verdosas en esas dos especies, pero siempre como un elemento parcial y reducido de su coloración rojiza de fondo. Lepidochitona rufoi puede distinguirse de las otras especies en el Caribe por su coloración crema claro con marcas irregulares ocre amarillo. Cromáticamente, L. rufoi puede compararse con ejemplares de $L$. beanii Carpenter, 1857, de la Provincia Panámica. Lepidochitona beanii es de coloración variada, se reportan ejemplares color crema con manchas oscuras (Kaas \& Van Belle 1985).

La valva anal de L. rufoi, vista dorsalmente, tiene una forma más rectangular, menos circular que la de las otras dos especies caribeñas. La valva anal del Holotipo de $L$. rosea tiene de ancho 1.8 veces el largo del tegumento; en $L$. liozonis la proporción es de 1.6 (según ilustradas en Kaas 1972). En L. rufoi la proporción de 2.5 más ancha que larga le da a esa valva una forma distinta.

La presencia de gránulos en la superficie del tegumento de $L$. rufoi es la característica que mejor la distingue de las otras especies de este género en el Caribe. La escultura del tegumento de L. liozonis como la de $L$. rosea es casi nula (Figs. 8A y B). La densidad de estetos (totales) de L. rufoi (4 000/ $\mathrm{mm}^{2}$ en promedio, $\mathrm{n}=2)$ es menor a la encontrada en L. liozonis, (5 200 a $\left.6500 / \mathrm{mm}^{2}, \mathrm{n}=3\right)$ y en L. rosea $(7350$ a 


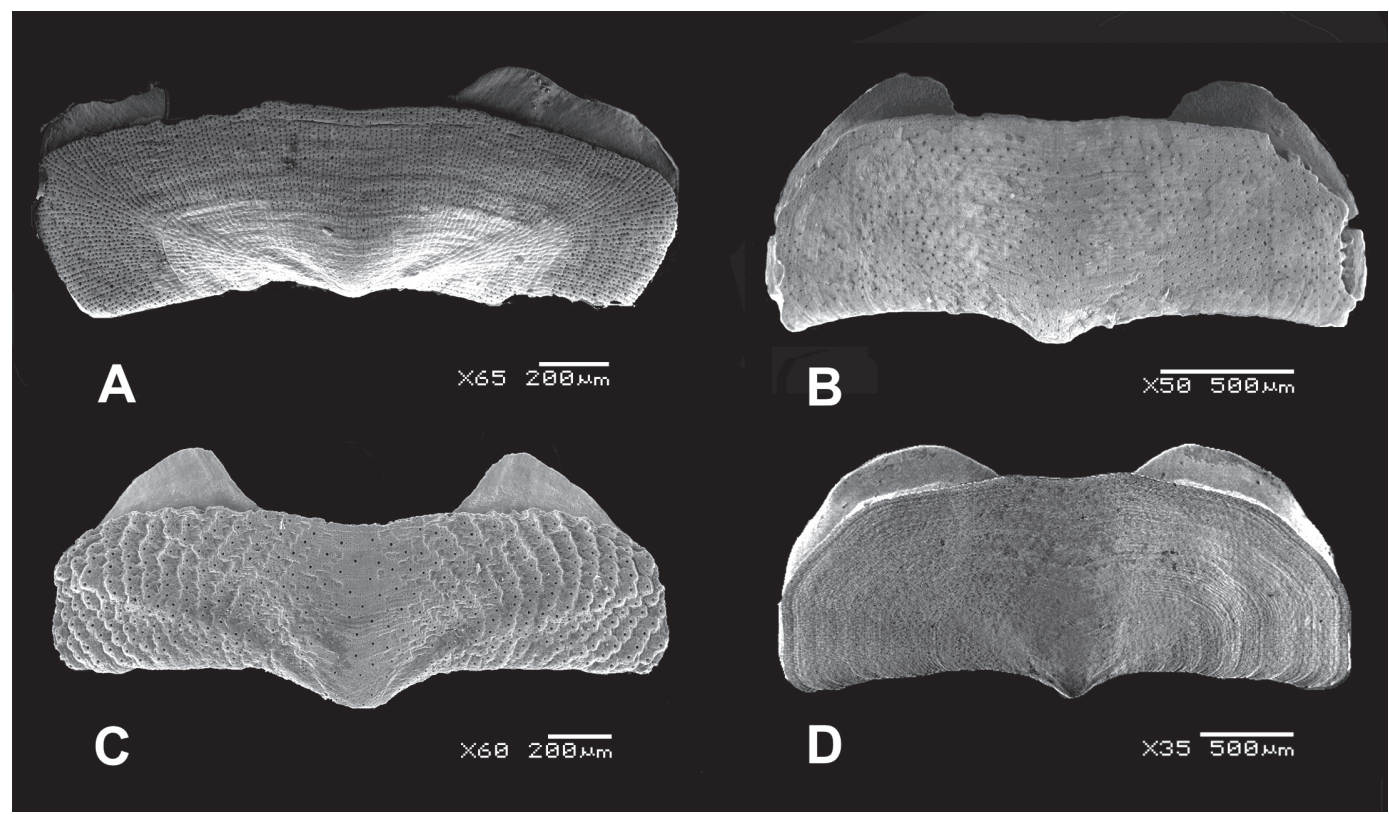

Fig. 8. Valva IV de: A, Lepidochitona liozonis (Dall \& Simpson, 1901), CBUPRH \#2002; B, L. rosea Kaas, 1972, CBUPRH \#2435; C, L. beanii Carpenter, 1857, CBUPRH \#2076; D, L. salvadorensis García-Ríos, 2006, CBUPRH \#2049.

Fig. 8. Valve IV: A, Lepidochitona liozonis (Dall \& Simpson, 1901) CBUPRH \#2002; B, L. rosea Kaas, 1972, CBUPRH \#2435; C, L. beanii Carpenter, 1857, CBUPRH \#2076; D, L. salvadorensis García-Ríos, 2006, CBUPRH \#2049.

$8500 / \mathrm{mm}^{2}, \mathrm{n}=2$ ). Esta comparación preliminar requiere una muestra mayor para determinar si es significativa.

El número de hendiduras en la valva anal de L. rufoi (9) es menor que las de L. liozonis (10-11) y las de L. rosea (10-12); sin embargo, este criterio puede ser variable, estos resultados se obtuvieron a partir de las observaciones realizadas a dos valvas. Los análisis del número de hendiduras de las valvas ha demostrado ser un criterio débil para la clasificación a nivel de especie (Eernisse et al. 2007). Las espículas marginales del cinturón de esta especie son similares a las descritas por Kaas (1972) para L. rosea y presentan el doble de la longitud de las de L. liozonis. Los corpúsculos dorsales de L. rosea y L. liozonis duplican el diámetro de los de L. rufoi.

La rádula de $L$. rufoi presenta el diente central estrecho, mientras que en L. liozonis este diente es cuatro veces más ancho (García-Ríos 2003) y tres veces más ancho en L. rosea.
Los trabajos taxonómicos utilizando la bioquímica comparada sugieren la existencia de tres clados dentro de la familia Lepidochitonidae (Eernisse 2004). En esa reagrupación se reconoce la cercanía de los lepidoquitones del Caribe con los de la zona Panámica, poblaciones que quedaron aisladas en el Plioceno medio, cuando emergió el Istmo de Panamá. Reconociendo los pares o grupos de especies muy similares, que están actualmente aislados, Ferreira (1985) propuso que la "especie hermana" de L. liozonis era L. beanii. Con la descripción de esta nueva especie e incluyendo otra Lepidochitona de reciente descripción (GarcíaRíos 2006), podemos actualizar la propuesta de Ferreira. Lepidochitona beanii es la especie hermana de L. rufoi (Fig. 8), considerando que ambas poseen el tegumento microgranuloso. Lepidochitona salvadorensis García-Ríos, 2006 (Panámica) es la especie con mayor similitud a L. liozonis y L. rosea, las tres con tegumentos de superficies relativamente lisas. 


\section{AGRADECIMIENTOS}

Una licencia sabática otorgada por la Universidad de Puerto Rico en Humacao me permitió completar este manuscrito. El microscopio electrónico de barrido fue prestado por el Programa Partnership for Research \& Education in Materials (PREM) de la Universidad de Puerto Rico-Humacao (NSF-DMR-0934195). Agradezco a Jesús E. Gómez Carrasquillo su esfuerzo extraordinario para obtener más ejemplares para esta descripción y a Migdalia Álvarez Ruiz por su participación en las recolectas en Tobago, de donde se obtuvo importante material de referencia.

\section{RESUMEN}

El género Lepidochitona Gray, 1821 tiene dos especies en el Caribe: L. liozonis (Dall \& Simpson, 1901) y L. rosea Kaas, 1972. Se describe una tercera especie: Lepidochitona rufoi sp. nov. de Puerto Rico. Lepidochitona rufoi difiere de ambas por tener un tegumento finamente granuloso, menor tamaño, una rádula con dientes centrales muy finos, la forma de la valva anal y el color diferentes. También difiere de L. liozonis por tener espículas marginales más largas.

Palabras clave: Mollusca, Polyplacophora, Lepidochitona, Puerto Rico, especie nueva.

\section{REFERENCIAS}

Abbott, R.T. 1974. American seashells. Van Nostrand Reinhold Company, Nueva York, EEUU.

Bullock, R.C. 1985. The Stenoplax limaciformis (Sowerby, 1832) species complex in the New World (Mollusca: Polyplacophora: Ischnochitonidae). The Veliger 27: 291-307.

Dall, W.H. \& C.T. Simpson. 1901. The Mollusca of Porto Rico. Bull. U.S. Fish Commission for 1900. 20: 351524, pls. 53-58.
Eernisse, D.J. 2004. Revival of the genus Cyanoplax Pilsbry, 1892 for a clade of West Coast chitons, p. 33-35. In J.C. Martínez \& R.V. Yeomans (eds.) Program and Abstracts of the $37^{\text {th }}$ Annual Meeting of the Western Society of Malacologists, Ensenada, Baja California, México.

Eernisse, D.J., R.N. Clark \& A. Draeger. 2007. Polyplacophora, p. 701-713. In J.T. Carlton (ed.). Light and Smith Manual: The intertidal invertebrates of Central California to Oregon. University of California, Berkeley, California, EEUU.

Ferreira, A.J. 1985. Chiton (Mollusca: Polyplacophora) fauna of Barbados, West Indies, with the description of a new species. Bull. Mar. Sci. 36: 189-219.

García-Ríos, C.I. 2003. Los quitones de Puerto Rico. Isla Negra Editores, San Juan, Puerto Rico.

García-Ríos, C.I. 2006. A new species of Lepidochitona (Mollusca: Polyplacophora) from El Salvador. The Veliger 48: 206-214.

García-Ríos, C.I. \& M. Álvarez-Ruiz. 2008. Los quitones de Tabago. Memorias XXVII Simposio de la flora y fauna del Caribe. Universidad de Puerto Rico en Humacao, Humacao, Puerto Rico.

Kaas, P. 972. Polyplacophora of the Caribbean region. Stud. Fauna Curaçao 4: 62, 247 figuras, 9 láminas.

Kaas, P. \& R.A. Van Belle. 1985. Monograph of living chitons (Mollusca: Polyplacophora).Vol. 2. Suborder Ischnochitonina: Ischnochitonidae: Schizoplacinae, Callochitoninae and Lepidochitoninae. E.J. Brill, Leiden, Holanda.

Kaas, P. \& R.A. Van Belle. 1998. Catalogue of living chitons (Mollusca: Polyplacophora). Backhuys, Leiden, Holanda.

Slieker, F.J.A. 2000. Chitons of the world, an illustrated synopsis of recent Polyplacophora. L'Informatore Piceno, Ancona, Italia.

Smithe, F.B. 1975. Naturalist's color guide. The American Museum of Natural History, Nueva York, EEUU. 